NOTA CIENTÍFICA

\title{
Reporte de albinismo en Podiceps major, Pelecanus thagus y Cinclodes fuscus y revisión de aves silvestres albinas del Perú
}

\author{
Report of albinism in Podiceps major, Pelecanus thagus y Cinclodes fuscus \\ and a revision of wild albino birds from Peru
}

\author{
Miriam Torres e Irma Franke
}

Departamento de Ornitología, Museo de Historia Natural, Uniunseo de Historia Natural, Uniersidad Nacional Mayor de San Marcos. Av. Arenales 1256, Jesús María, Apartado 14-0434, Lima, Perú.

Email Irma Franke:

irma.franke@gmail.com

$\begin{array}{ll}\text { Presentado: } & 14 / 09 / 2007 \\ \text { Aceptado: } & 08 / 02 / 2008\end{array}$ $\begin{array}{ll}\text { Aceptado: } & 08 / 02 / 2008 \\ \text { Publicado online: } & 21 / 07 / 2008\end{array}$

\begin{abstract}
Resumen
Reportamos observaciones de individuos albinos en tres especies de aves peruanas, el Zambullidor Grande (Podiceps major), el Pelícano peruano (Pelecanus thagus) y el Churrete cordillerano (Cinclodes fuscus). Los individuos eran albinos parciales casi totalmente blancos, aparentemente adultos, que mostraron un comportamiento normal entre sus conespecíficos. La supervivencia después de varios meses pudo ser comprobada para el Pelícano peruano y el Churrete cordillerano. Debido a que la información publicada sobre albinismo en aves peruanas es muy escasa, se realizó una revisión y se recopilaron registros para otras nueve especies que son también presentados.
\end{abstract}

\section{Abstract}

We report observations of albino individuals in three species of Peruvian birds, Great Grebe (Podiceps major), Peruvian Pelican (Pelecanus thagus) and Bar-winged Cinclodes (Cinclodes fuscus). These were all three mostly white partial albinos, apparently adult birds that showed normal behavior among normal conspecific individuals. Survival after several months was confirmed for the Peruvian Pelican and the Bar-winged Cinclodes. Since published information on albinism in Peruvian birds is very scarce, a revision was made and records for nine other species were compiled and are also presented.

Keywords: Albinism, Podiceps major, Pelecanus thagus, Cinclodes fuscus
La presencia de albinismo en algunas aves se da como consecuencia de una serie de mutaciones de genes que afectan la producción de melanina, esto origina la falta de pigmentación parcial o total en el plumaje y partes descubiertas. La mayor parte de registros de aves albinas provienen de Europa y Norteamérica, probablemente debido al alto número de observadores de aves que existen en esos lugares. Para el caso específico de Irlanda por ejemplo, existen más de 160 registros de aves que muestran albinismo parcial o total (BirdWatch Ireland, 2006). Lo contrario ocurre en Sudamérica, donde los reportes de estas aves son escasos, muy dispersos, muchos no están documentados y sólo en unos pocos casos han sido publicados. En el presente trabajo reportamos el avistamiento de individuos albinos de tres especies de aves en el Perú, Podiceps major, Pelecanus thagus y Cinclodes fuscus, incluyendo aspectos de la supervivencia de dos de ellas y hacemos una revisión de casos de albinismo de aves peruanas.

\section{Podiceps major (Boddaert, 1783); Zambullidor Grande}

El 10 de febrero de 2007, en la Laguna Ñapique (5³2'56” S; 80¹'53,62” W), provincia de Sechura, departamento de Piura, Perú, observamos un individuo de Zambullidor grande parcialmente albino (Fig. 1). Este ejemplar presentaba el pico y las patas claras y ojos de color oscuro. Las plumas del cuerpo eran de color blanco con manchas negruzcas en los extremos de ambas alas y parte baja del dorso. Este individuo fue observado nadando entre un grupo de zambullidores de coloración normal. Durante un rato prologado se separó del grupo y permaneció solo, para luego acercarse a otros individuos nuevamente. Este comportamiento fue observado también en individuos normales por lo que podría suponerse que no tiene problemas de socialización con los otros miembros de su misma especie. El ejemplar fue extensamente fotografiado.
Pelecanus thagus Molina, 1782; Pelícano Peruano

El 11 de julio de 2006, en la caleta de pescadores Puerto Rico (5॰48'47"S; 811'46,2”W) ubicada en la provincia de Sechura, departamento de Piura, Perú, observamos cerca al muelle un Pelícano peruano blanco (Fig. 2). Este individuo presentó ausencia de pigmentos obscuros en sus partes descubiertas: pico y patas. La cabeza, cuello y dorso presentaron plumas marrones claras y el vientre y las alas plumas blancas. La presencia de plumas ligeramente marrones puede ser una señal de reducción de pigmentación o leucismo no muy marcado. El 9 de febrero del 2007, siete meses después, se regresó a la caleta Puerto Rico y en el muelle se observó nuevamente a este Pelecanus thagus (Fig.3). Al igual que en la época anterior este individuo se encontraba solo. Pescadores locales contaron que este individuo era observado usualmente solitario y era favorecido por la población del puerto, quienes le proporcionaban alimento favoreciendo así su supervivencia. Se obtuvo documentación fotográfica de ambas observaciones.

\section{Cinclodes fuscus (Vieillot, 1818); Churrete Cordillerano}

El 22 de setiembre de 2006, en el pueblo de Brea (7339'16,5” S, 154'50,2” W, $3650 \mathrm{~m}$ de altitud) ubicado en la provincia de Parinacochas, departamento de Ayacucho - Perú, encontramos un Cinclodes fuscus parcialmente albino (Fig. 4). El individuo fue fotografiado en un área abierta de pajonal rocoso y se encontraba sólo en el momento de la observación. Este Churrete cordillerano presentaba todas las plumas del cuerpo blancas, ausencia de pigmentación en el pico y patas y los ojos negros. El 3 de marzo de 2007, 5 meses y medio después, tuvimos la oportunidad de regresar nuevamente al pueblo de Brea, y pudimos observarlo nuevamente en una zona no muy alejada a la de la primera observación (Fig. 5). 


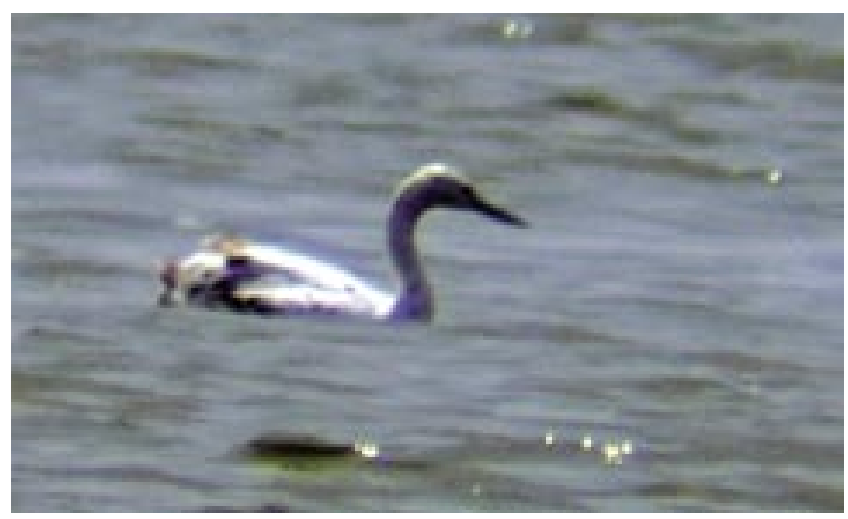

Figura 1. Podiceps major (Boddaert, 1783) albino.

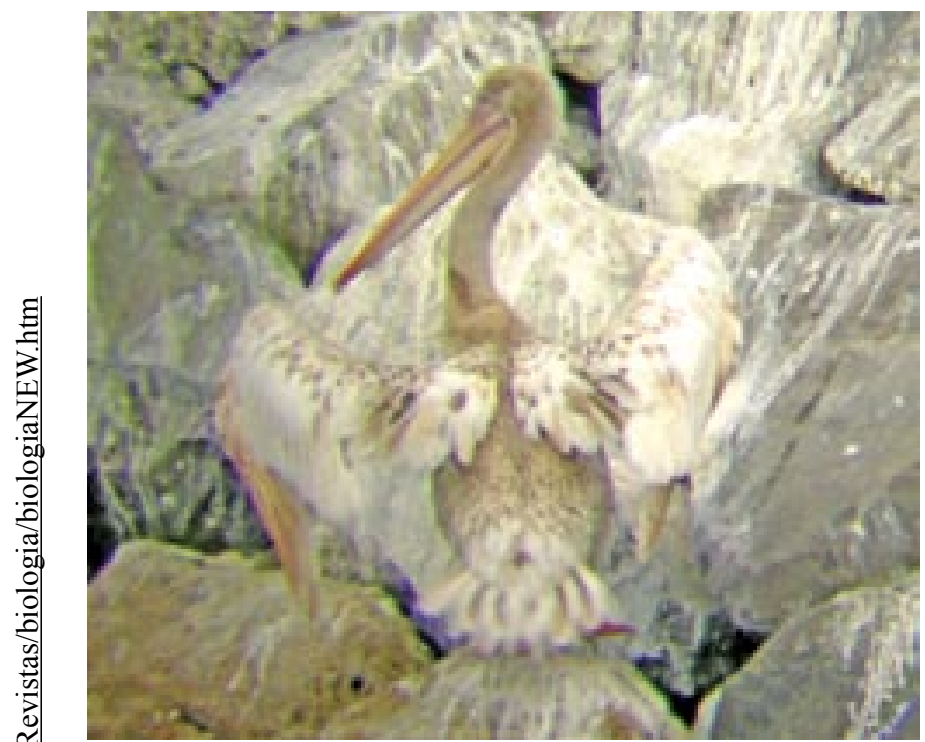

Figura 3. Pelecanus thagus Molina albino, 09/02/2007.

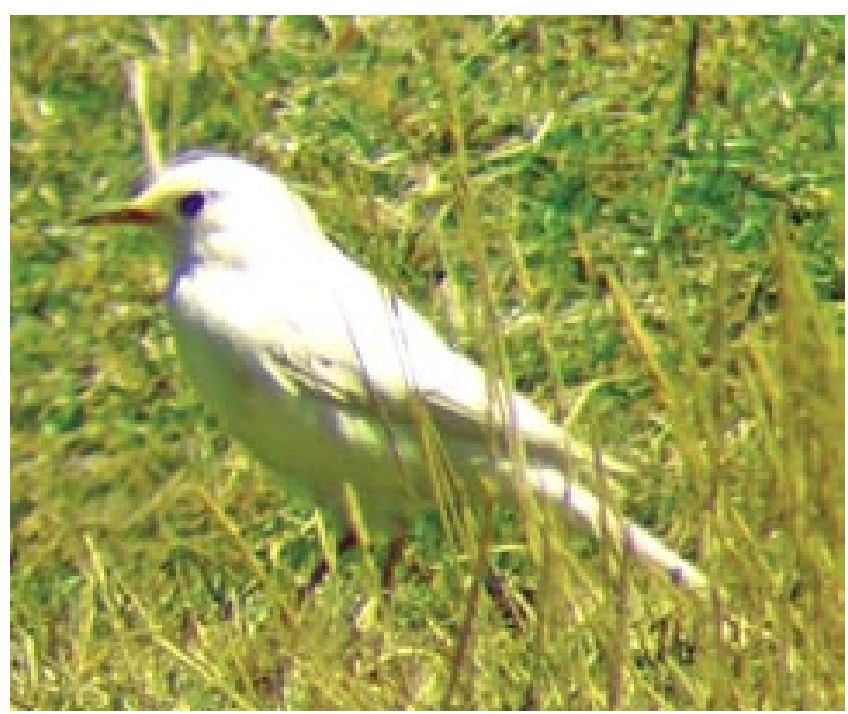

Figura 5. Cinclodes fuscus (Vieillot, 1818) albino, 03/03/2007.

En esta ocasión el individuo albino se desplazaba entre el pajonal acompañado de un individuo de coloración normal. Sin embargo ningún comportamiento observado permite afirmar si era su pareja. Se obtuvo documentación fotográfica de ambas observaciones.

Las principales dificultades que enfrentan los individuos albinos son principalmente problemas intraespecíficos, con sus congéneres, y enfermedades debido a la intensidad de la luz tales

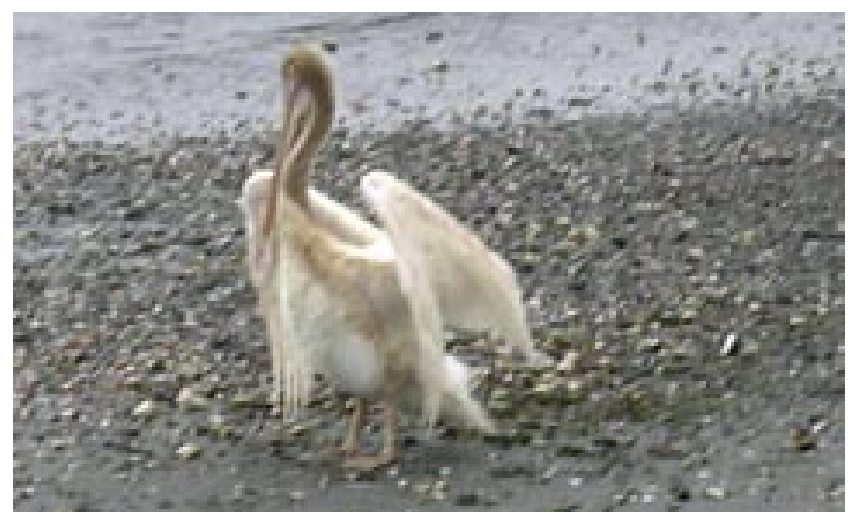

Figura 2. Pelecanus thagus Molina albino, 11/07/2007.

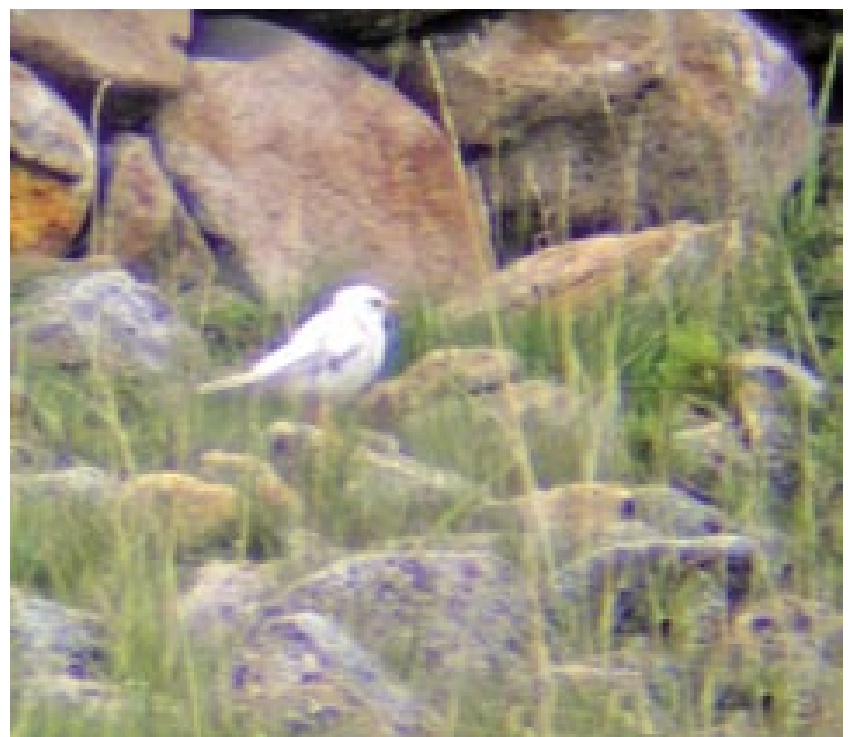

Figura 4. Cinclodes fuscus (Vieillot, 1818) albino, 22/09/2006.

como la baja visibilidad al igual que problemas con los rayos ultravioleta, así como también ser fácilmente visibles por sus depredadores. En los tres casos reportados, Podiceps major, Pelecanus thagus y Cinclodes fuscus, los tamaños y comportamiento de los individuos nos permiten presumir que se trataría de individuos adultos. Esto nos sugiere que estas aves se desenvolverían sin grandes dificultades en sus hábitats, es decir, que su alimentación al igual que protección no se vería afectada a pesar de su inusual color. A pesar de ello, su éxito reproductivo y su supervivencia podrían verse afectados por su coloración.

Si bien es cierto que la observación de aves silvestres con algún tipo de albinismo no es muy común, es de esperar que se hayan realizado un cierto número de avistamientos. Una revisión de publicaciones sobre aves peruanas y otras fuentes de información permitió reunir reportes de individuos albinos de otras nueve especies. La difusión de estos avistamientos es sin embargo muy pobre. Sólo para dos especies existen publicaciones. Para las otras cuatro especies, fotografías de los individuos albinos fueron difundidas a través de páginas Web y para una especie se realizaron discusiones por Internet. En un caso existen ejemplares en colecciones ornitológicas y otro caso está documentado mediante fotografías no publicadas. Debido a la exigua información publicada existente sobre casos de albinismo en las aves peruanas hemos reunido toda aquella disponible sobre los individuos conocidos, que son descritos a continuación. 


\section{Larus modestus Tschudi, 1843; Gaviota Gris}

Christian Alvarez observó un ejemplar leucístico de esta gaviota en la Playa Sarapampa, provincia de Cañete, departamento de Lima el 27 de febrero de 2006. El individuo presentaba el pico negro, ojos oscuros y patas rojizas. Las plumas de la cabeza, dorso y vientre eran de color blanco con algunas plumas de color marrón claro. Las plumas primarias y cobertoras primarias y secundarias de ambas alas eran de color marrón más oscuro. Se han publicado fotos de este individuo en Birdingperu (2007).

\section{Plegadis ridgwayi (Allen, 1876); Yanavico}

Juan Chalco observó un Yanavico parcialmente albino en marzo del 2006 en el Lago de Junín, departamento de Junín. Esto concuerda con la observación realizada por Fjeldsa, según la cual el mayor número de ejemplares albinos de esta especie se encuentran en el Lago de Junín (Fjeldså y Krabbe, 1990). Esta ave se caracterizaba por presentar casi todo su plumaje blanco con algunas manchas oscuras en el ala y cuello. Sin embargo tanto pico como patas eran de color negro. Se publicaron fotos de este individuo en BirdingPeru (2007).

Arturo Carlos también observó individuos albinos de esta especie. Entre Abril de 2006 y abril de 2007 registró 4 individuos parcialmente albinos en humedales del departamento de Lima: Laguna el Paraíso, Humedales de Puerto Viejo y Pantanos de Villa. Estos individuos presentaban plumas blancas en el dorso y en un caso debajo de las alas. El ejemplar fue fotografiado (com.pers.)

\section{Elaenia gigas Sclater, 1871; Elainia moteada}

Yábar y Yábar (2004) reportaron el avistamiento en enero de 2004 de un individuo parcialmente albino en Amazonia Lodge, ubicado en el Parque Nacional del Manu, departamento de Madre de Dios. Este ejemplar presentaba el pico rosado, iris oscuro y patas negras. Las plumas del cuerpo eran de color blanco con algunas manchas de color crema. Fotografías de esta ave han sido publicadas en Birdingperu (2007).

\section{Sayornis nigricans (Swainson, 1827); Mosquero de Agua}

Fernando Angulo reportó la observación de un ejemplar parcialmente albino en setiembre de 2006 en Laquipampa, departamento de Lambayeque (com. pers.). Este ejemplar presentaba plumas blancas en todo el cuerpo a excepción de la corona, la cual era de color negro. El iris al igual que el pico tenían coloración oscura, mientras que las patas eran de color claro. Fotografías de este individuo han sido publicadas en Birdingperu (2007).

\section{Pyrocephalus rubinus (Boddaert, 1783); Turtupilín}

Ortiz de la Puente colectó en agosto de 1927 dos ejemplares de esta especie con albinismo parcial en el departamento de Lima. El primer individuo presentaba albinismo en el dorso, plumas terciarias de ambas alas, plumas cobertoras de las alas y algunas de la cola. El segundo individuo presentaba albinismo en las plumas secundarias de ambas alas y plumas cobertoras de las primarias.
Ambos ejemplares fueron colectados y se encuentran en la colección ornitológica del Museo de Historia Natural - San Marcos (MUSM 4148; MUSM 4150).

Mimus Iongicaudatus Tschudi, 1844; Calandria de Cola Larga

Fernando Angulo menciona el avistamiento de un ejemplar albino probablemente total en la localidad de Olmos, departamento de Lambayeque en julio del 2004 (com. pers.). No existe documentación de este caso.

Turdus chiguanco Lafresnaye \& D'Orbigny, 1837; Zorzal Chiguanco

Esta es la especie de la que se ha reportado el mayor número de casos de albinismo parcial. Todos los ejemplares fueron reportados en noviembre de 2005 en varios departamentos del sur del Perú. Mauricio Ugarte reportó el avistamiento de un ejemplar en el departamento de Puno del que no se especifica localidad; Christian Albujar reportó el avistamiento de otro ejemplar en la localidad de Yura en Arequipa y Berioska Quispe reporta el avistamiento de otro ejemplar en la localidad de Cora y Sicuani en el departamento de Cusco. Ellos señalan que el albinismo parcial presente en estas aves se observó en las plumas de la cola y cabeza (BirdingPeru, 2007). No se dispone de información sobre registros fotográficos para esta especie.

\section{Oreomanes fraseri Sclater, 1860; Picocono Gigante}

Servat (2002) reportó un ejemplar con albinismo parcial en el departamento de Lima en Noviembre de 1997. Este individuo presentaba el pico y patas negras y el iris de coloración oscura. El plumaje del dorso y cuello eran de color rufo y en la parte inferior del vientre habían plumas blancas.

\section{Dacnis sp. (Thraupidae); Dacnis}

Dan Lane (com. pers.) observó un ejemplar totalmente blanco en el Río Pauya, departamento de Loreto en agosto del 2000. Este ejemplar no fue colectado ni documentado.

De los casos de albinismo reportados y recopilados, la mayoría se refiere a aves paseriformes terrestres, con el mayor número en los Mosqueros (Tyrannidae). Sólo tres avistamientos conciernen a aves acuáticas. La información disponible, sin embargo, no permite deducir si esto se debe a oportunidad de observación, a la frecuencia de casos de albinismo o a la difusión de la información. De estos casos conocidos, las especies peruanas para las que se conoce mayor número de individuos albinos son el Yanavico, Plegadis ridwagi, y el Zorzal Chiguanco, Turdus chiguanco.

\section{Agradecimientos}

Un agradecimiento especial a Dan F. Lane por su aporte y ayuda en la elaboración de este artículo. Así mismo, un agradecimiento a todas las personas mencionadas en este artículo las cuales nos permitieron utilizar su información.

\section{Literatura citada}

Birdingperu 2007. Pics \& Files. < http://www.birdingperu.com/picsfiles/>. Acceso 23/07/07 
BirdWatch Ireland 2006. Albinism in birds and other unusual plumage types. Information Leaflet $\mathrm{N}^{0} 5$. <http://www. birdwatchireland.ie/bwi/pages092003/ learnaboutbirds. html $>$. Acceso 23/07/07.

Fjeldså, J. \& Krabbe, N. (1990) The birds of the high Andes. Copenhagen: Zoological Museum, University of Copenhagen \& Svendborg: Apollo Books.

Servat, G. (2002) A case of partial albinism in Giant Conebill - Oreomanes fraseri. Cotinga 18:80

Yábar R. y V. Yábar. (2004) An albino Mottle - backed Elaenia Elaenia gigas in Manu Biosphere Reserve, Perú. Cotinga 22:106 